\title{
Designing Chatbots as Community-Owned Agents
}

\author{
Michal Luria \\ Carnegie Mellon University \\ Pittsburgh, PA, USA \\ mluria@cs.cmu.edu
}

\author{
Joseph Seering \\ Carnegie Mellon University \\ Pittsburgh, PA, USA \\ jseering@cs.cmu.edu
}

\author{
Jodi Forlizzi \\ Carnegie Mellon University \\ Pittsburgh, PA, USA \\ forlizzi@cs.cmu.edu
}

\author{
John Zimmerman \\ Carnegie Mellon University \\ Pittsburgh, PA, USA \\ johnz@cs.cmu.edu
}

\begin{abstract}
This work investigates how social agents can be designed to create a sense of ownership over them within a group of users. Social agents, such as conversational agents and chatbots, currently interact with people in impersonal, isolated, and often one-on-one interactions: one user and one agent. This is likely to change as agents become more socially sophisticated and integrated in social fabrics. Previous research has indicated that understanding who owns an agent can assist in creating expectations and understanding who an agent is accountable to within a group. We present findings from a three week case-study in which we implemented a chatbot that was successful in creating a sense of collective ownership within a community. We discuss the design choices that led to this outcome and implications for social agent design.
\end{abstract}

\section{CCS CONCEPTS}

- Human-centered computing $\rightarrow$ Human computer interaction (HCI); User centered design; User studies.

\section{KEYWORDS}

Conversational agents, Chatbots, Ownership, Interaction Design

\section{ACM Reference Format:}

Michal Luria, Joseph Seering, Jodi Forlizzi, and John Zimmerman. 2020. Designing Chatbots as Community-Owned Agents. In 2nd Conference on Conversational User Interfaces (CUI '20), July 22-24, 2020, Bilbao, Spain. ACM, New York, NY, USA, 3 pages. https://doi.org/10.1145/3405755.3406134

\section{INTRODUCTION}

People routinely interact with social agents such as conversational agents, chatbots and robots, but interactions with them are impersonal, single instances. Unlike other technologies, the design of current conversational agents (CUIs) does not explicitly support or communicate neither personal nor shared ownership of agents-all users are treated equally, but rarely as unique individuals.

Previous research has shown that this may need to be addressed by design as CUIs become more socially sophisticated and personalized [6]. For instance, when an individual asks for access to their partner's calendar, should the CUI enable it? When two people have contradicting requests, how should the agent respond? Understanding who an agent belongs to, and as a result who it is accountable to, can assist in setting expectations for CUI behavior.

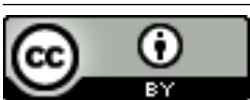

This work is licensed under a Creative Commons Attribution International 4.0 License.

CUI '20, fuly 22-24, 2020, Bilbao, Spain

(C) 2020 Copyright held by the owner/author(s).

ACM ISBN 978-1-4503-7544-3/20/07.

https://doi.org/10.1145/3405755.3406134
While CUIs are on a trajectory towards understanding social context and responding accordingly [1], designers do not yet know how to design social technology to communicate ownership. One of the first questions designers may want to address is whether a CUI equally belongs to all members of a group, e.g., a family (following an "appliance model" [3]), or whether it is personally owned by an individual (following a "profile model" [3]).

Previous research has examined device sharing behaviors [7], and how they reflect on people's relationships [5]. However, not much work has looked into what it means to mutually own and use a social agent. According to Self Extension theory, physical artifacts are not merely owned by individuals, but can be a critical component in shaping their self perception [2]. Furthermore, possessions can make individuals feel that they belong to a group or community [4].

As an initial exploration on this space, we created and tested a social chatbot for Twitch, an online game-streaming community. In a three week long implementation, the bot was successful in creating a sense of mutual ownership within the community [9]. Due to its success, we retrospectively analyzed the design choices and community responses, and describe three choices that contributed to the perception of shared ownership over it. We reflect on resulting implications for users, designers and service providers.

\section{THE DESIGN OF BABYBOT}

In a field study fully described in [9], we implemented a chatbot in an established online Twitch community. This community was run by a "streamer," who livestreamed himself played games, and included about 20-30 regularly active members. The chatbot, named BabyBot (and later renamed "PeteBot"), was intended to "be raised" within the community. BabyBot was designed as part of an effort to introduce conversational chatbots that integrate in a larger social environment, beyond dyadic interaction [8]. Its goal within this community was to generate new interactions between users, to support moderation, and to encourage users to reflect on their community [9].

Over the course of three weeks, the bot "grew" while interacting in the channel. The bot's behavior was created through rules-based and Markov-chain based text generation, combined with predefined behaviors for each "life stage" (full technical description in [9]). Using this design, the bot's language corpus was based only on what individuals said in the chat throughout the study period, and resulted in the generation of 1154 bot messages. This was a simple technical approach based on a small corpus (approximately 1000 chatroom messages when the bot began generating text). However, the goal of this work was to test a design, rather than a technical, approach. 


\section{COMMUNITY OWNERSHIP OF AN AGENT}

In our implementation we found that the narrative of "raising a bot" and its interaction with the community were successful in indicating shared ownership. We describe three key design choices that we believe contributed to this perception.

\subsection{Creation of the Agent}

Self Extension theory suggests that creation is key in building a sense of ownership and extending oneself onto an object [2]. We designed BabyBot with the explicit intent to allow users to participate in its creation over time. The first part of this design approach involved "learning" features-community members were told that BabyBot would learn from the community, and that their conversations in the channel would shape how it "grew up." As soon as BabyBot began using words in its "toddler phase," community members also began seeing their role in shaping its behavior.

Some of the most engaging moments happened when members of the community recognized pieces of the bot's generated text as something they or someone else had previously said. This behavior was particularly gratifying when community members had tried to "teach" the bot something, as in the quote below where one user had mischievously tried to teach the bot to "enjoy" vodka. We believe this capacity to teach a CUI, even in a very simple, whimsical way, is a significant contributor to creating a sense of individual ownership, and to the acceptance of the bot as part of the community:

$$
\text { baby_bot_some vodka thinking } 、(\cdot \forall \cdot) \text { ) }
$$$$
\text { P2: I'm so proud :'-( }
$$

Users were able to determine the bot's identity in a broader sense as well. Halfway into the study, the community expressed a desire to rename BabyBot, as it was no longer a baby. After running a poll and discussing options, the community decided to name the bot "PeteBot." In order to accommodate this, the researchers created a new Twitch account and swapped the login credentials in the bot's script. The bot was also designed with a fairly light "backstory," which allowed users to speculate about its origins, habits, and motivations, and to create their own narratives to fill in these gaps.

\subsection{Personal Responsibility over the Agent}

The community regularly discussed whether they were "raising" the bot well or not. Users frequently expressed humorous concern that they were doing so poorly when it picked up "bad behavior" such as saying age-inappropriate things or expressing violence. In reaction to "bad parenting" by some users, others attempted to "fix" the bot through playfully strict interaction:

$$
\begin{aligned}
& \text { pete_bot_can hit so } \mathrm{o}(>\omega<) \mathrm{o} \\
& \text { P8: @pete_bot_don't hit things! } \\
& \text { P10: @pete_bot_Bad pete } \\
& \text { pete_bot_: @P10 }(\wedge \vee \forall \wedge) \\
& \text { P11: @pete_bot_bad bot, no hit so@_@ }
\end{aligned}
$$

Users shared the understanding that their own interaction with the bot influenced its behavior, and that as a result they all shared the responsibility of "raising it" right. We believe that such interaction, that promotes personal alongside collective responsibility, was also a contributing factor to the community's sense of agent ownership.

\subsection{Attention to Individuals}

In addition to group interactions, several instances of personal interaction with a single user were key in the process of accepting the bot. While in our study these instances were somewhat incidental, they should be considered for intentional design in the future. In the example below, BabyBot interacted with $\mathrm{P} 1$, who is frequently teased within the community for filling the role of the community's "mother." BabyBot captured this notion, which resulted in a strong reaction from $\mathrm{P} 1$, and perhaps a stronger personal connection. More importantly, the interaction seemed to have created a sense of individual familiarity and personalized interaction that contributed to the community's overall sense of ownership:

P1: Tell me more, @pete_bot_ pete_bot_: @P1 is nobodys mom of any kind $\mathrm{o}(>\omega<) \mathrm{O}$ P1: YES! Pete knows what's up!

P1: Still struggling with the apostrophes, but whatever. Streamer: HA! P1 just got what she's been fighting for since the moment she entered this channel, the first time she was ever here, this is the moment she has been waiting for. Congratulations P1, it looks like you have been set free.

\section{CONCLUSION}

BabyBot was successful in integrating within this online community and in engaging its members. Over the course of the study, the community developed interaction habits with the bot that mirrored the ones they had with each other (like saying hello and goodnight to it), and expressed their acceptance of the bot by gifting it a subscription to the channel that only regular members had.

In this paper we present several design strategies that we believe contributed to creating a sense of ownership over the bot within the community: (1) enabling users to creatively shape it, (2) promoting a sense of individual responsibility, and (3) creating opportunities for personal interactions.

However, this kind of community-based ownership would be more complicated for current CUI providers and their users-service providers are the ones who "own" agents, have control over them, and collect and make use of personal and interactional data. This may prevent a sense of community ownership over an agent in real-life implementation. In order to design for more than simply the false sense of ownership, service providers may need to shift their service design from creating agents that are tied to the brand, to agents that can be shared and co-created within a community.

\section{ACKNOWLEDGMENTS}

We would like to thank our collaborators Connie Ye, Jessica Hammer and Geoff Kaufman, and our participants on the Twitch platform.

\section{REFERENCES}

[1] Amazon. accessed September 9, 2019. Making Alexa More FrictionFree. https://developer.amazon.com/blogs/alexa/post/60e1f011-3236-4162-b0f6$509205 \mathrm{~d} 354 \mathrm{ca} /$ making-alexa-more-friction-free

[2] Russell W Belk. 1988. Possessions and the extended self. fournal of consumer research 15, 2 (1988), 139-168.

[3] AJ Bernheim Brush and Kori M Inkpen. 2007. Yours, mine and ours? Sharing and use of technology in domestic environments. In International Conference on Ubiquitous Computing. Springer, 109-126.

[4] Mihaly Csikszentmihalyi and Eugene Halton. 1981. The meaning of things: Domestic symbols and the self. Cambridge University Press. 
[5] Jane Gruning and Siân Lindley. 2016. Things we own together: Sharing possessions at home. In Proceedings of the 2016 CHI Conference on Human Factors in Computing Systems. ACM.

[6] Michal Luria, Rebecca Zheng, Bennett Huffman, Huang Shuangni, John Zimmerman, and Jodi Forlizzi. 2020. Social Boundaries for Personal Agents in the Interpersonal Space of the Home. In Proceedings of the 2020 CHI Conference on Human Factors in Computing Systems. ACM.

[7] Tara Matthews, Kerwell Liao, Anna Turner, Marianne Berkovich, Robert Reeder and Sunny Consolvo. 2016. She'll just grab any device that's closer: A Study of Everyday Device \& Account Sharing in Households. In Proceedings of the 2016 CHI Conference on Human Factors in Computing Systems. ACM, 5921-5932.
[8] Joseph Seering, Michal Luria, Geoff Kaufman, and Jessica Hammer. 2019. Beyond Dyadic Interactions: Considering Chatbots As Community Members. In Proceedings of the 2019 CHI Conference on Human Factors in Computing Systems (Glasgow, Scotland Uk) (CHI '19). ACM, New York, NY, USA, Article 450, 13 pages. https://doi.org/10.1145/3290605.3300680

[9] Joseph Seering, Michal Luria, Connie Ye, Geoff Kaufman, and Jessica Hammer. 2020. It Takes a Village: Integrating an Adaptive Chatbot into an Online Gaming Community. In Proceedings of the $2020 \mathrm{CHI}$ Conference on Human Factors in Computing Systems (Honolulu, Hawaii, USA) (CHI '20). ACM, New York, NY, USA, Article 579, 13 pages. https://doi.org/10.1145/3313831.3376708 